\title{
Protein docking prediction using predicted protein-protein interface
}

\author{
Bin $\mathrm{Li}^{1}$ and Daisuke Kihara ${ }^{2,1,3^{*}}$
}

\begin{abstract}
Background: Many important cellular processes are carried out by protein complexes. To provide physical pictures of interacting proteins, many computational protein-protein prediction methods have been developed in the past. However, it is still difficult to identify the correct docking complex structure within top ranks among alternative conformations.

Results: We present a novel protein docking algorithm that utilizes imperfect protein-protein binding interface prediction for guiding protein docking. Since the accuracy of protein binding site prediction varies depending on cases, the challenge is to develop a method which does not deteriorate but improves docking results by using a binding site prediction which may not be 100\% accurate. The algorithm, named PI-LZerD (using Predicted Interface with Local 3D Zernike descriptor-based Docking algorithm), is based on a pair wise protein docking prediction algorithm, LZerD, which we have developed earlier. PI-LZerD starts from performing docking prediction using the provided protein-protein binding interface prediction as constraints, which is followed by the second round of docking with updated docking interface information to further improve docking conformation. Benchmark results on bound and unbound cases show that PI-LZerD consistently improves the docking prediction accuracy as compared with docking without using binding site prediction or using the binding site prediction as post-filtering.

Conclusion: We have developed PI-LZerD, a pairwise docking algorithm, which uses imperfect protein-protein binding interface prediction to improve docking accuracy. PI-LZerD consistently showed better prediction accuracy over alternative methods in the series of benchmark experiments including docking using actual docking interface site predictions as well as unbound docking cases.
\end{abstract}

Keywords: protein docking prediction, protein-protein interaction, interaction site prediction

\section{Background}

Many important cellular processes, such as gene expression regulation and transport, are carried out by protein complexes [1-3]. The importance and the abundance of protein interactions and complexes have been recently further highlighted by large-scale protein-protein interaction maps revealed for many organisms [4-7]. The tertiary structure of proteins is necessary for understanding the underlying molecular mechanism of protein interaction [2], however, it is often difficult to obtain complex structures by experimental methods, e.g. the X-ray crystallography or NMR. Thus, experimentally solved protein complex structures only share a small fraction

\footnotetext{
* Correspondence: dkihara@purdue.edu

${ }^{2}$ Department of Biological Science, Purdue University, West Lafayette, IN, 47907, USA

Full list of author information is available at the end of the article
}

among known protein complexes confirmed by biochemical experiments. Therefore, an important task in bioinformatics is to develop efficient and accurate computational methods for predicting protein-protein docking conformations.

Many protein-protein docking methods have been developed in the past employing various ideas and techniques [8-20]. Typically a docking prediction for a pair of proteins produces a few thousands of docking conformations (docking decoys), which are subject to ranking using a scoring function. Conformational search algorithms employed include the Fast Fourier Transform (FFT) $[16,17,21]$, the Geometry Hashing [18,22], Monte Carlo algorithms [13], genetic algorithm [23,24], and Langevin dynamics [25]. For scoring a docking decoy, usually several terms are combined, which include physics-based scores [26] and those concern geometrical
C Biomed Central

C 2012 Li and Kihara; licensee BioMed Central Ltd. This is an Open Access article distributed under the terms of the Creative Commons Attribution License (http://creativecommons.org/licenses/by/2.0), which permits unrestricted use, distribution, and reproduction in any medium, provided the original work is properly cited. 
shape complementarity $[18,27,28]$. Clustering of docking decoys is also shown to be effective in selecting near native conformations [29-31]. Some of the recent docking algorithms have more elaborate procedures, for example, by considering alternative conformations of flexible protein chains [32] or post docking optimization steps $[14,33]$. Nevertheless, despite significant efforts of developing methods, it is still difficult to identify and rank the correct conformations in top ranks among hundreds of decoys $[18,27,34]$ as is also evidenced by results from recent Critical Assessment of Prediction of Interactions (CAPRI), a community wide experiment on the comparative evaluation of protein-protein docking methods [10].

The accuracy of docking prediction could improve when a part, even if not all, of protein-protein interface (PPI) residues are known. PPI residues for a pair of interacting proteins can be identified by experiments including point mutation such as the alanine scanning [35-38], chemical modification of residues [39,40], NMR [41], hydrogen/deuterium exchange [42], and disulfide cross-linking [43]. If several PPI residues are known, they can be simply used for filtering, i.e. to select docking decoys which have the known PPI residues at their docking interface $[44,45]$. Alternatively, known PPI residues from interacting proteins can be incorporated as distant constraints [14]. However, experimental methods are time consuming. This is particularly true if identification of a whole PPI region of an interacting protein pair is attempted or if investigating many interacting proteins in a network is planned.

PPI residues can be also predicted by computational methods, which capture sequence and structural features of PPI regions [46]. There are a number of PPI site prediction methods developed. Sequence features used for PPI site prediction include amino acid residue propensity [46-52], sequence conservation [53-57], and correlated mutation [58-60]. Structure information used include hydrophobic patches, the secondary structure propensity [51], atom group propensity [61], relative accessible surface area [47], geometrical surface shape [47], the crystallographic B-factor [51], and energetic characteristics of PPI residues $[62,63]$. Current protein interface prediction methods choose one or combinations of these features to construct scoring functions for machine learning techniques [51,55,56,64-67]. Recent development of PPI site prediction methods has been overviewed in recent review articles [68,69]. The obvious advantage of the computational methods over experimental methods is that the former can be performed much faster than the latter. However, the problem of computational prediction methods is that they are not always accurate. For example, the Meta-PPISP method [70], one of the state-of-the-art methods, predicts PPI residues on average with a precision of $50 \%$ at the coverage of $50 \%$ for enzyme-inhibitor complexes [71]. Moreover, the prediction accuracy varies depending on target proteins and thus it is difficult to estimate the accuracy for individual cases. Therefore, computational PPI residue prediction cannot be reliably used for simple post-filtering of docking decoys. A naive use of PPI residue prediction for post-filtering may actually decrease the prediction accuracy, as we will show in Results.

Here, we present a novel protein docking algorithm, PI-LZerD (using Predicted Interface with Local 3D Zernike descriptor-based Docking algorithm), which utilizes imperfect PPI residue prediction for guiding proteinprotein docking. PI-LZerD performs iterative improvement of docking results starting from an initial run of docking that uses potentially inaccurate PPI prediction as restraints. The base of the docking algorithm used is the LZerD (Local 3D Zernike descriptor-based Docking algorithm), which we have developed previously [18]. The idea of using additional predicted information for aiding protein docking has been explored by a few previous works. In their works, PPI information is used for post-filtering docking decoys [16,71-73] or incorporated as an additional scoring term $[14,45,74,75]$. Compared to these related works, the current work is significantly different in the design and some important aspects: First, we have developed a novel algorithm which is specifically designed to utilize imperfect PPI prediction. Thus, we don't use PPI information simply for post filtering. Second, we perform thorough investigation on how the accuracy of PPI prediction affects to the docking prediction accuracy. PI-LZerD is shown to be able to consistently improve docking predictions when actual PPI predictions are used for unbound docking cases. The datasets used and the developed PI-LZerD program are made freely available for academic community.

\section{Methods}

\section{Pairwise protein docking algorithm, LZerD: the original algorithm}

We start with brief explanation of the original LZerD pairwise protein-protein docking algorithm [18]. As will be explained in the next section, PI-LZerD performs an iterative use of a modified version of LZerD. LZerD takes two protein tertiary structures (Protein Data Bank, PDB [76], files) as input (termed a ligand and a receptor protein) and outputs over 30000 50000 of docking decoys ranked by a scoring function. The geometric hashing algorithm [77] is used for docking conformational search.

Given a protein tertiary structure, protein surface is constructed and then points are distributed evenly on the surface. Typically, about 1500 2000 points are distributed for a 200-250 amino acid long protein (Figure 1A). The geometric hashing procedure pre-computes 


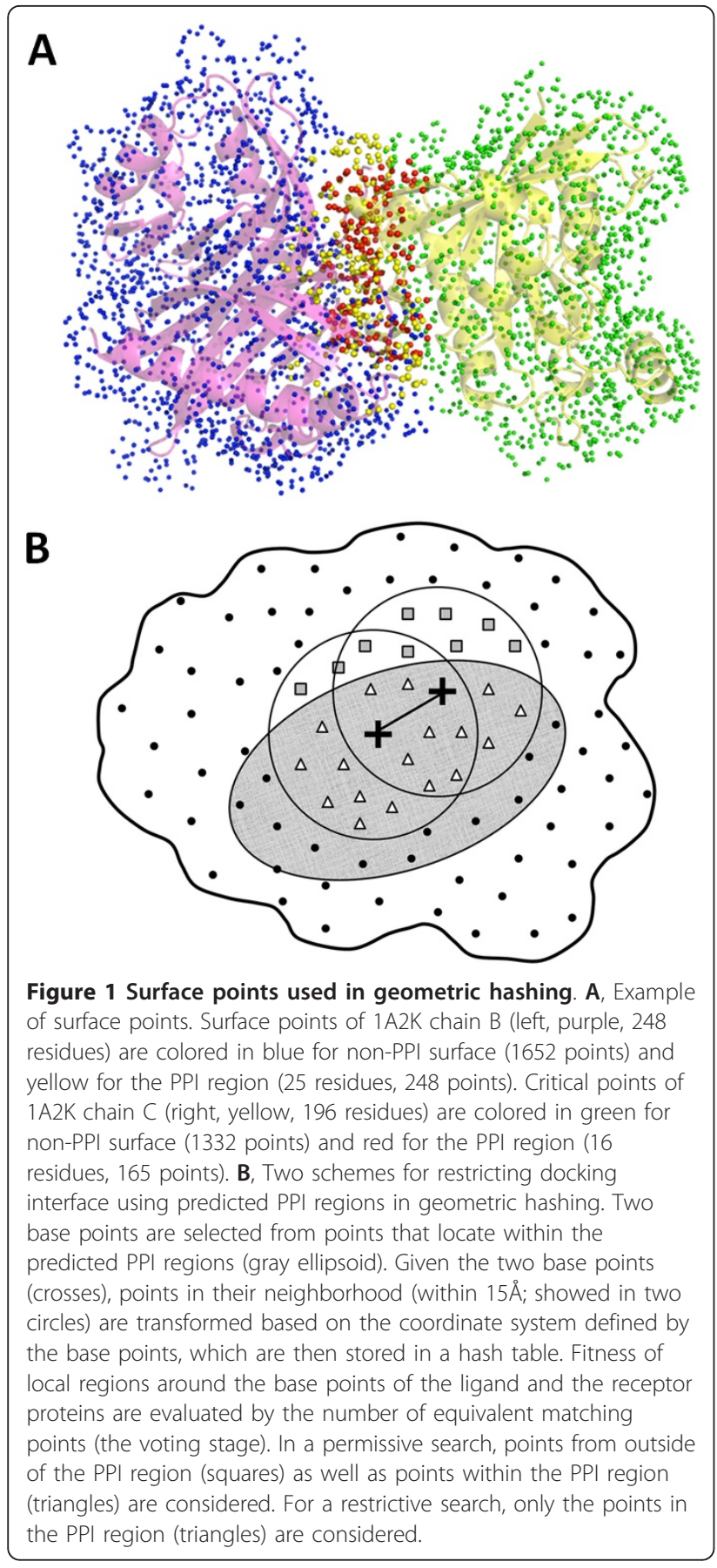

and records all possible orientations of the ligand protein (the hashing stage). This is done by defining a coordinate system for each pair of surface points (called base points), based on which the coordinates of the other neighboring surface points (within $15 \AA$ to the base points) are computed. Note that a three dimensional coordinate system can be uniquely defined by two points by using the average of normal vectors of the two points as additional parameter. Once all the poses of the ligand protein are recorded in a hash table, each possible pose of the receptor protein is computed in the same way, which are then compared with the poses of the ligand protein stored in the hash table (the matching stage). If a sufficient number of points from the two proteins match (voting stage), the conformation is further evaluated by a physics-based score.

The scoring function is a weighted sum of the following terms: van der Waals, where, repulsive and attractive parts of the term are considered separately [13]; an electrostatics term, which considers repulsive/ attractive and short-range/long-range contributions separately [78]; a hydrogen and disulfide bond term [79]; two solvation terms [80,81]; and a knowledgebased atom contact term [82]. Weighting factors for the linear combination of the terms were trained on two datasets, the protein-protein docking benchmark 2.0 [83], which contains 84 pairwise unbound-unbound and bound-unbound docking structures, and also on 851 protein-protein dimeric complexes compiled by Huang and Zou [84]. The combination of weight values were determined by using logistic regression with the interface root mean square deviation (iRMSD) between predicted decoys and the native structure as the target function to be optimized.

\section{Modified LZerD to incorporate PPI prediction}

We modified the LZerD algorithm so that additional information of a PPI region can be used to restrict the docking search space. Figure 1B illustrates the two methods of restricting conformational search space in geometric hashing. Given a set of (predicted) PPI residues in a ligand or a receptor protein, each surface point is classified into either PPI (points within the gray ellipsoid in Figure 1B) or non-PPI depending on whether the closest atom for the point belong to a PPI residue or not. In the geometry hashing, two base points (two crosses) are selected to define a reference coordinate system, based on which the other local points are transformed. Base points are selected only from the PPI surface points for both ligand and receptor proteins. Then, in the voting stage, matching points between the ligand and receptor are counted either only from the PPI surface points (i.e. matches are only considered within the predicted PPI regions; triangles in the region in gray in Figure 1B) or from all the surface points (triangles and squares) including non-PPI points. Obviously, the former seeks for a geometrical complementarity of the two proteins only at the predicted PPI regions while the latter explores a wider surface area to identify well fitting docking conformation in the vicinity of the predicted PPI regions. PI-LZerD uses these two search areas in different stages of docking conformation search. The more permissive search area is considered 
for the initial LZerD run and the more restricted searches are performed for the subsequent iterations.

\section{PI-LZerD algorithm}

The PI-LZerD algorithm performs pairwise protein-protein docking prediction using additional information of PPI residues as constraints. The algorithm is illustrated in Figure 2. First, given the tertiary structure and (predicted) PPI regions of the two proteins to be docked, the modified LZerD is run to yield typically 30000 50000 docking decoys (the right branch of the diagram). For this initial run of the modified LZerD, all neighboring surface points to the base points are considered in the voting stage (the permissive search as discussed in the previous section). The docking decoys are ranked by the physics-based score and top 1000 best scoring decoys are selected.

The 1000 decoys are subject to clustering by considering the similarity of docking interface regions. For a given pair of docking decoys, common atoms between the two PPI regions from the two decoys are selected. Then, the RMSD is computed for the common atoms only when the common atoms share more than $60 \%$ of all interface atoms of both PPI sites (if the common atoms do not exceed $60 \%$ then the two proteins are not clustered together). We call it the common interface RMSD (ciRMSD) of two docking decoys. The ciRMSD is more suitable for the PI-LZerD algorithm as compared to the conventional coordinate RMSD [85] or the ligand RMSD [86], since it focuses on capturing the similarity of docking interface regions.

Once the ciRMSD is computed for all the pairs of decoys, 60 decoys are selected by considering the physics-based score and the cluster size of the decoys. First, the decoy with the lowest score (the lower, the better) is selected and close decoys with a ciRMSD $\leq 4.0 \AA$ are discarded from the pool. This process is repeated until 30 decoys are identified. Next, additional 30 decoys are selected based on the cluster size. For each of the decoys, the number of the other decoys within $4.0 \AA$ ciRMSD is computed. Then, the largest cluster (i.e. the center decoy with the largest number of close decoys) is selected. If several clusters with the same size are found, the one which has the center decoy with the lowest physics-based score is selected. All the decoys in the cluster are removed, and the process is repeated until 30 representative decoys are selected. Consequently, 30 decoys are selected based on the lowest energy and 30 more decoys are selected based on the cluster size. It was shown that combining the energy value and the cluster size can find more hits than using a single metric alone (Additional file 1, Figure S1).

The selected 60 decoys are passed to the subsequent process. For each of the 60 docking decoys, PPI residues are extracted. PPI residues are defined as those which have a heavy atom closer than $5.0 \AA$ to any atom to the docking partner. The decoys do not necessarily have the identical PPI region as the initially provided PPI information because the modified LZerD has explored the vicinity of the input PPI in the docking conformation search. Using the identified PPI residues as the updated constraint, the modified LZerD is run for the second time. In this round, only the PPI surface points are considered at the voting stage in the geometric hashing (the restrictive search). From the resulting docking decoys, the top 1000 lowest energy docking decoys are clustered based on ciRMSD, whose cluster centers are sorted by the physics-based score. Since the modified LZerD is run for each of the 60 decoys, in total of 60 LZerD runs are performed.

In addition to the 60 runs of the modified LZerD, we run the original LZerD without using predicted PPI information followed by post-filtering by using the predicted PPI residues (naive-filtering method) (the left branch of Figure 2). In the naive-filtering method, docking decoys are sorted not by the physics-based score but by the agreement of the docking interface residues to the predicted PPI residues. Therefore, the overall procedure produces 61 runs of docking predictions, i.e. 61 ranked lists of docking decoys. To make the final ranking of docking decoys, first, the top ranked docking decoys from each of the 61 lists are ranked by the physics-based score, and then the decoys in the same subsequent ranks from the 61 lists are ranked in the same way. Thus, the decoys from all the lists are first sorted by their ranks in each list then sorted by the physicsbased score. If the identical decoys appear, one which is ranked lower in the entire final list is removed (it is not common but possible that identical docking decoys appear in different LZerD runs).

\section{Dataset of protein complexes and PPI information}

The first dataset we use for benchmarking PI-LZerD is the protein-protein docking benchmark version 3.0 [87] with 124 bound cases. The average length of the proteins is 256 and the number of docking interface residues of the proteins range from 10 to 70 with an average of 25 .

To investigate how the accuracy of PPI prediction affects to the docking prediction, we first use "simulated" PPI predictions as input. The actual PPI region of a ligand and a receptor proteins are shifted by $5,10,12$, and 15 residues to two opposite directions on the protein surface along the major axis of the PPI region. To shift a PPI region on the surface, $n$ PPI residues $(n=5$, $10,12,15)$ at one end of the PPI site along the axis are removed and the same number of residues are added on the opposite side of the PPI site. Thus, the shifting of 


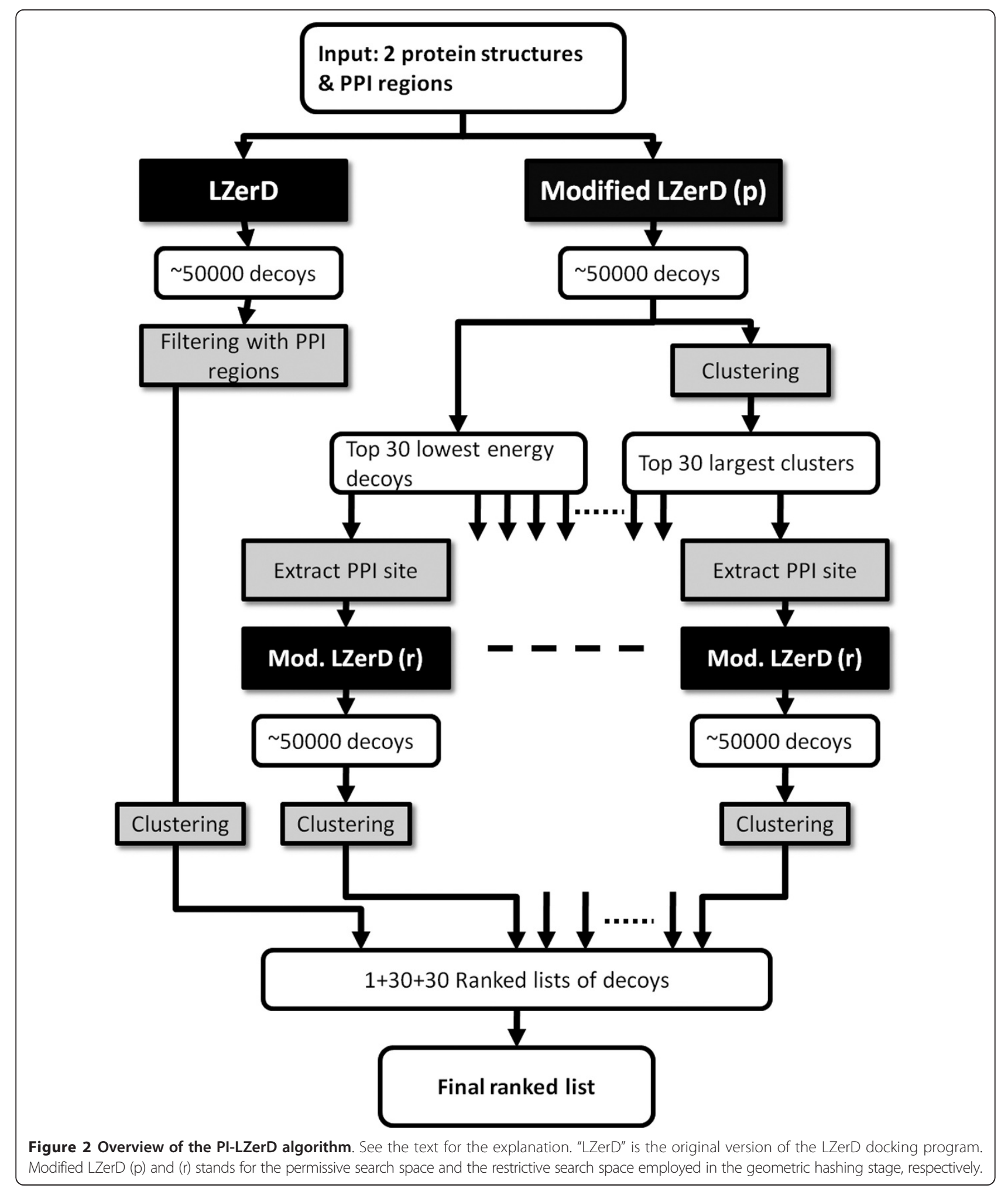

PPI regions are done geometrically rather than along the protein sequence (Additional file 1, Figure S2). By combining two shifted PPI regions from a ligand and a receptor protein, four test cases are made for each protein complex (because the PPI region on each protein is shifted in two opposite directions). The protein complexes are removed from the dataset if one of proteins has a smaller PPI region than the number of 
shifted residues. The total number of tested protein complexes with $5,10,12$, and 15 PPI residues shift are $124(124 \times 4=496$ test cases), 122 (488 cases), 118 (472 cases), and 104 (416 cases), respectively. Since four different combinations of shifted PPI regions of a ligand and a receptor are tested, the number of tested cases is four times of the number of protein complexes, which is shown in the parentheses.

Figure 3A shows the distribution of the sensitivity of shifted PPI regions (i.e. the fraction of the correctly predicted PPI residues among actual PPI residues). The sensitivity depends on the size of the proteins PPI regions for the same number of residues shifted. The average sensitivity for the $5,10,12$, and 15 residue shifted PPI sites are $0.767,0.535,0.447$, and 0.324 , respectively. The specificity value (the fraction of correctly predicted PPI residues over the total number of predicted PPI residues) is the same as the sensitivity because the size of a shifted PPI region is the same as the actual one. In Figure 3B, the fraction of correctly interacting PPI residue pairs in the protein complexes with shifted PPI regions is shown (the fraction of the native contacts, fnat [88]). The fnat value depends on the shifting directions even for the same pair of shifted PPI sites. The average fnat value for proteins pairs with 5 residue shifted PPI is 0.673 . The fnat value distributes more broadly for more shifted PPI sites. The average PPI sites for 10, 12, 15 shifted residues are $0.364,0.275$, and 0.191 .

We also test PI-LZerD using actual PPI predictions with a state-of-the-art PPI prediction method, metaPPISP [70]. Meta-PPISP is a meta server which combines predictions by three methods, Promate [51], PINUP [49], and cons-PPISP [54]. The benchmark dataset is selected from the iPFAM database [89], a subset of PFAM database [90], which provides multiple sequence alignments (MSA) of interacting proteins. We used iPFAM because meta-PPISP needs a MSA as an input. The iPFAM entries were pruned using the following criterion: (1) PFAM families with 20 to 100 seed sequences were selected. (2) PFAM families consisting local domain sequences were replaced with their corresponding full-length sequences from UniProt [91]. A representative PDB structure was then selected from each PFAM family given by the association in iPFAM. (3) Protein structures that do not have any observable interacting partners in their PDB files were removed. (4) Proteins with their PDB entries that have non-standard amino acids and obsolete PDB files were filtered out. (4) PDB structures with antibody-antigen and proteinDNA/RNA interactions were removed. (5) Protein complexes with more than two chains are removed. (6) Complexes were eliminated if they are classified as monomers bound by crystal contacts in the PQS
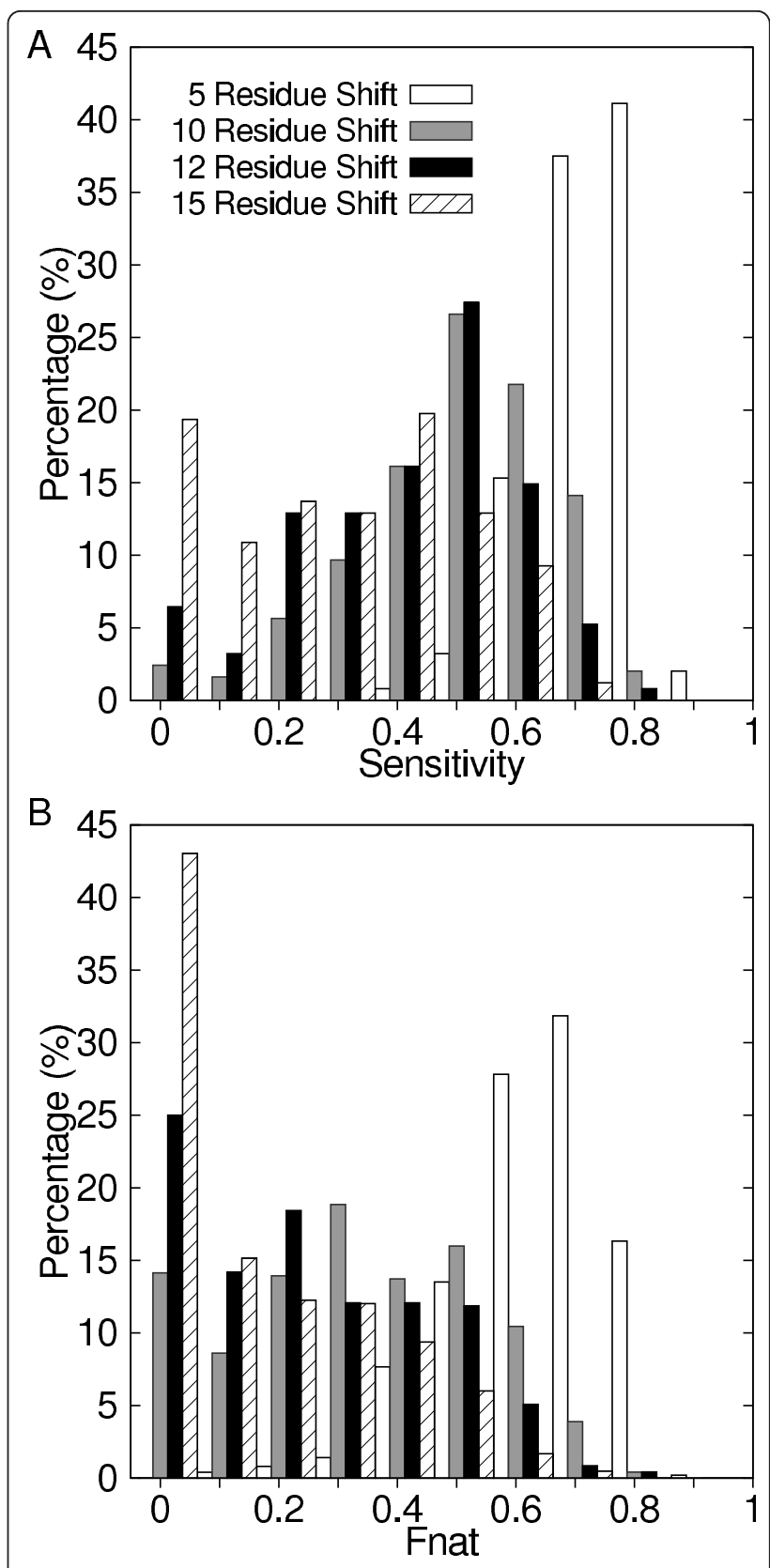

Figure 3 Accuracy of the shifted PPI regions. Distributions of $\mathbf{A}$, the sensitivity (= specificity); $\mathbf{B}$, the fnat value; of the shifted PPI regions by $5,10,12$, and 15 residues.

definition [92]. (7) Proteins with the size between 75 to 300 amino acids were selected. (8) In the final dataset, PFAM families with redundant representative structures with $\geq 35 \%$ sequence identity were filtered out. Given that MSAs in PFAM may not have the PDB structure as a part of the alignment, we employed MUSCLE (ver. 3.6) [93] with default parameters to compute MSAs from PFAM unaligned sequences and one sequence from the selected PDB structure. The final dataset 
includes 127 protein complexes. Using prediction output of the meta-PPISP server, residues which have a metaPPISP score of 0.1 or higher are identified as PPI residues.

\section{Availability and requirements}

The executable program of PI-LZerD for Linux is freely available to academic institutions at our website, http:// kiharalab.org/PI-LZerD. The datasets used in this study are also available at the same webpage. The program requires a computer with at least 1.5 GB RAM operated by Linux OS. The average times combining both docking and scoring range are about 1-2 hours for small proteins (about 400 points on the receptor and ligand) and it may take longer for larger proteins. This timing is reported on a computer with a dual-core $2.1 \mathrm{GHz}$ processor with 8 GB RAM. In addition, the pairwise docking program, LZerD, which is the base of PI-LZerD, is also made available at http://kiharalab.org/ proteindocking.

\section{Results and Discussion}

\section{Naive post-filtering method}

An obvious approach to use predicted PPI information for protein docking prediction is to select docking decoys with a PPI site that agrees well to the provided PPI information. This approach, termed as the naive post-filtering method, was tested on datasets with the five different accuracy levels of PPI prediction. In addition to the set of accurate PPI information, we used PPI sites shifted by $5,10,12$, and 15 residues. For each protein complex with PPI information, we run original LZerD to produce top 1000 scoring docking decoys. Then, for each docking decoy, the fraction of the overlap of residues in the provided PPI information the PPI region of the docking decoy is computed for both ligand and for the receptor proteins, and the average of the two are used for sorting decoys.

In Figure 4, the fraction of the protein complexes where correct prediction (interface RMSD $\leq 2.5 \AA$ in Figure $4 \mathrm{~A} ; 4.0 \AA$ in Figure $4 \mathrm{~B}$ ) exist within specified ranks cutoff (x-axis) are shown. The prediction accuracy of original LZerD without using the PPI information is also shown for comparison. The naive post-filtering achieved near perfect prediction accuracy when the perfectly accurate PPI information was provided. When the PPI information was shifted by five residues, the prediction accuracy at top 1 rank dropped significantly, from $90 \%$ to $51 \%$ when iRMSD of $2.5 \AA$ is used as the threshold (Figure 4A). Interestingly, using further deteriorated PPI information of ten residues shift made prediction results indistinguishable from running LZerD without PPI information. Using more inaccurate PPI prediction of twelve or fifteen residue shifts was shown to be even

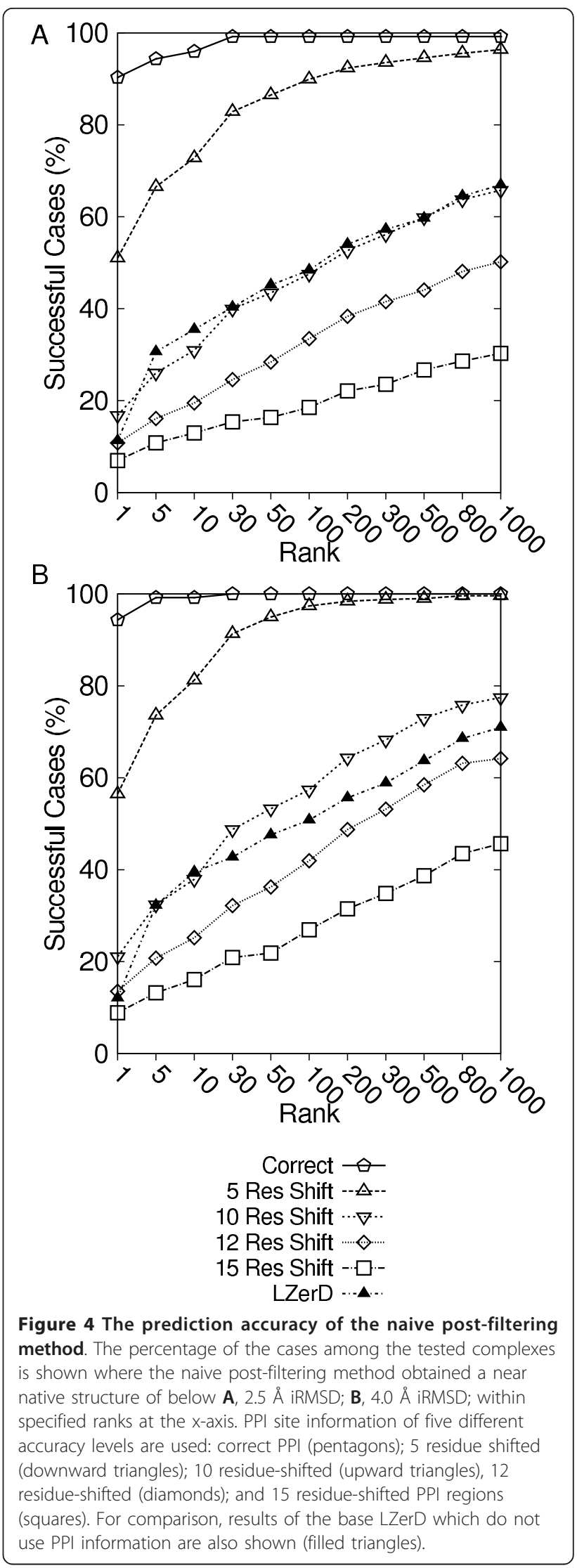


harmful, producing worse predictions than LZerD without PPI information. The consistent trend was observed in Figures $4 \mathrm{~A}$ and $4 \mathrm{~B}$. Overall, the results show that the naive post-filtering is sensitive to the accuracy of PPI residue information used for the filtering. Thus, it is not reliable to apply PPI prediction simply for post-processing when the accuracy of the prediction is not well known.

\section{PI-LZerD with simulated PPI predictions}

Next we examine performance of PI-LZerD on the dataset of simulated PPI predictions. This experiment is for understanding the effect of various levels of inaccuracy in PPI predictions to the docking results. In the later sections we discuss the results using actual PPI predictions on bound and unbound docking cases. The full implementation of PI-LZerD (Figure 2, PI-LzerD-2) was compared with four other variations of LZerD, namely, the original LZerD without PPI information (the base LZerD), the original LZerD followed by post-clustering without using PPI information, LZerD with naive postfiltering with the PPI information, and PI-LZerD using PPI information with only one iteration of the modified LzerD (PI-LZerD-1). PI-LZerD-1 clusters output of docking decoys using the ciRMSD.

Figure 5 shows prediction results of the five methods using $0,5,10,12$, and 15 residue shifted PPI information. The $y$-axis shows the fraction of successful cases where a correct prediction exists within specified ranks cutoff on the $\mathrm{x}$-axis. When the provided PPI residues are $100 \%$ accurate, the naive post-filtering can naturally select correct predictions among the pool of docking decoys (Figures 5A \&5B). PI-LZerD with one or two iterations performs better than the base LZerD. Since PI-LZerD does not restrict the conformation search space to the provided PPI site but also explores its neighborhood, PI-LZerD obtained a hit for a less number of complexes within top 30 ranks than the naive post-filtering method. However, when top 100 ranks are considered, both naive post-filtering and PI-LZerD-2 and PI-LZerD-1 made successful prediction for almost all the tested cases. The clustering step made a slight improvement of accuracy when applied to decoys generated by the base LZerD.

As the accuracy of the PPI information starts to deteriorate, the docking prediction accuracy by the naive post-filtering quickly drops relative to the others. When 5 residue shifted PPI information was used, the post-filtering method still showed the highest number of successful cases up to the 100 ranks (Figures 5C \&5D). When PPI regions were further shifted by 10 residues, PI-LZerD clearly outperformed the post-filtering method. The performance of the post-filtering method went down as low as the base LZerD which did not use the PPI information. It is also noticed that the PILZerD-2 performed better than PI-LZerD-1.

Figures $5 \mathrm{G} \& 5 \mathrm{H}$ show that when the 12 residue shifted PPI regions were used, the naive filtering method performed even worse than the base LZerD. In contrast, remarkably, PI-LZerD-2 managed to successfully use the inaccurate PPI information, showing a higher accuracy than the base LZerD. The accuracy of PI-LZerD-1 is now comparable to the base LZerD when $2.5 \AA$ iRMSD threshold was used (Figure $5 \mathrm{G}$ ) but better for $4.0 \AA$ iRMSD threshold (Figure 5H). Finally, with 15 residue shifted PPI regions (Figures 5I \&5J) PI-LZerD-2 still remained superior to the base LZerD while the accuracy by the naive post-filtering went further down. It is worth mentioning that the prediction accuracy by PILZerD-2 stays almost the same with 5, 10, 12, and 15 shifted PPI regions. Importantly, the stability of the prediction by PI-LZerD was observed only for PI-LZerD-2 but not PI-LZerD-1. This indicates that the two iterations of modified LZerD run are necessary to effectively explore the vicinity of specified PPI region to find the lowest energy conformation.

In Additional file 1, Figures S3 and S4, we analyzed the same results by classifying the shifted PPI sites by their accuracy. In Additional file 1, Figure S3, the protein complexes are classified by the average sensitivity of the shifted PPI sites of the receptor and the ligand proteins, while they are classified based on the fnat of shifted PPI sites of the receptor and the ligand proteins in Additional file 1, Figure S4. Essentially the same trend was observed in Additional file 1, Figures S3 \& S4 as Figure 5. Using the naive post-filtering, near perfect prediction accuracy can be achieved only when the correct PPI information is provided. However, its results quickly deteriorate as the accuracy PPI site information drops. In contrast, PI-LZerD can take advantage of PPI information even when it is not very accurate. For the range of the PPI site information accuracy tested, PILZerD always showed better performance than the base LZerD without using PPI information. It is very important that employing additional information (in this case PPI site prediction) do not deteriorate prediction results even if the quality of information is not high, which is successfully achieved by PI-LZerD.

\section{Docking Prediction using actual PPI site prediction}

Next, we use actual PPI site prediction for PI-LZerD. 127 protein complexes taken from the iPFAM database were used in this experiment. PPI site predictions were computed by Meta-PPISP [70] using MSAs taken from the iPFAM database. The average sensitivity and the specificity of the prediction by meta-PPISP were 0.648 and 0.297 , respectively (Figures $6 \mathrm{~A} \& 6 \mathrm{~B}$ ), when the cutoff score of 0.1 was used. The average sensitivity value 

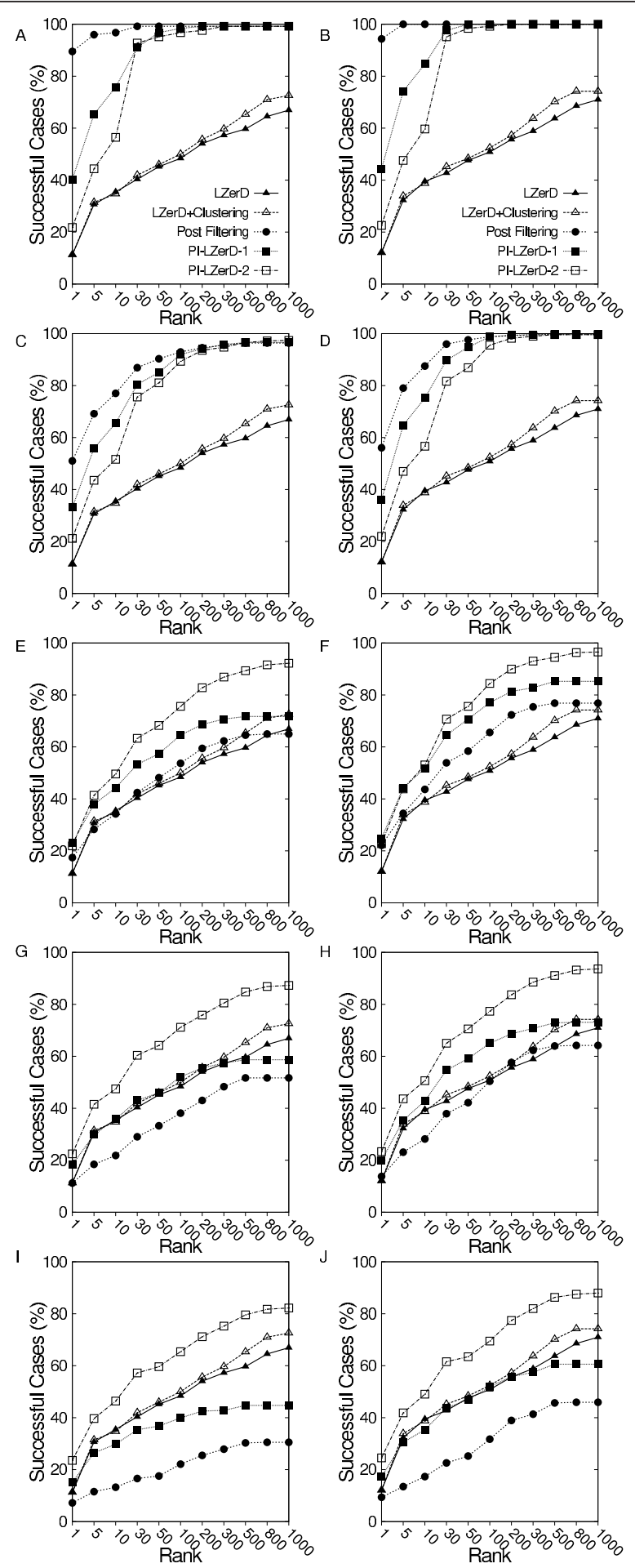

Figure 5 Docking prediction with simulated protein interface predictions. Four other methods are listed to compare with PI-LZerD-2: the base LZerD (LZerD), LZerD with clustering using ciRMSD (LZerD+Clustering), LZerD with one interaction of modified LZerD (PI-LZerD-1), and Simple residue filtering method (post-filtering). The $x$-axis indicates the ranks in logarithmic scale and the $y$-axis shows the percentage of cases where correct predictions are ranked equal or better than the corresponding ranks. Left panels, A, C, E, G, I, use the $2.5 \AA$ as threshold for correct predictions, while right panels, $\mathbf{B}, \mathbf{D}, \mathbf{F}, \mathbf{H}, \mathbf{J}$, use $4.0 \AA$ as the cutoff for near hit predictions. $\mathbf{A} \& \mathbf{B}$ use the correct protein interface information; $\mathbf{C} / \mathbf{D}$ use the simulated predictions with 5 residue shifts, $\mathbf{E} / \mathbf{F}, \mathbf{G} / \mathbf{H}$, and $\mathbf{I} / \mathbf{J}$ use the simulated predictions with 10, 12 , and 15 residue shifts, respectively. 


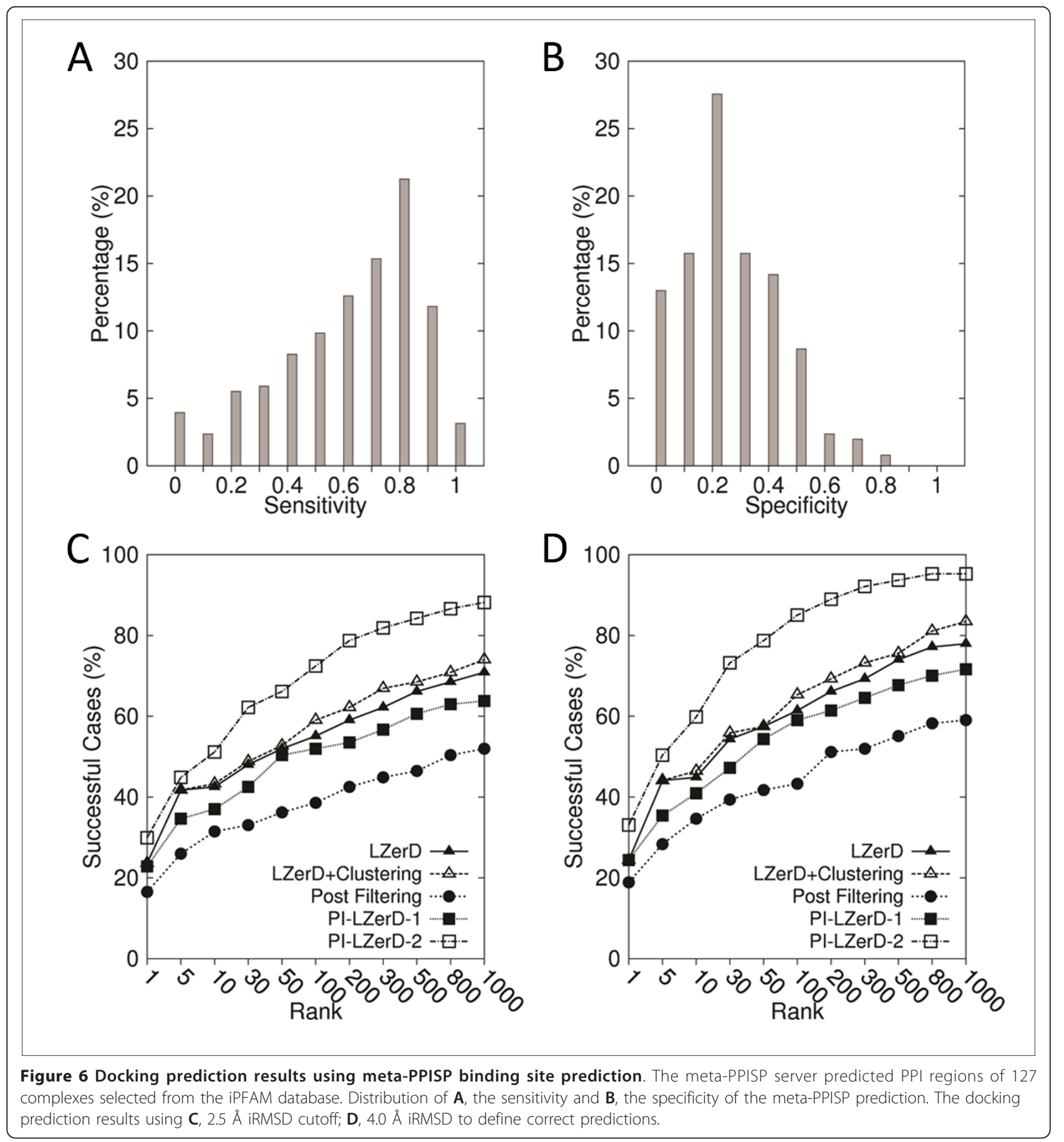

is better while the specificity value is worse than the simulated PPI site predictions we used in the previous section, which were 0.535 for both sensitivity and the specificity.

On this dataset, PI-LZerD-2 performed consistently the best at every rank cutoff ( $\mathrm{x}$-axis) with both $2.5 \AA$ and $4.0 \AA$ (Figures $6 \mathrm{C} \& 6 \mathrm{D}$ ) iRMSD thresholds. Within top 10 predictions, PI-LZerD-2 made at least one hit for
$51.2 \%$ of the cases, while the base LZerD and the naive post-filtering obtained hits for $42.5 \%, 31.5 \%$ of the cases with the $2.5 \AA$ iRMSD cutoff (Figure 6C). Within the rank of 100, the successful cases for the methods increased to $72.4,55.1$, and $38.6 \%$, respectively. Thus, PI-LzerD-2 improved the success rate over the base LZerD by 8.7 and $17.3 \%$ points within the rank of 10 and 100 . When $4.0 \AA$ is used for iRMSD cutoff (Figure 
6D), PI-LZerD-2 obtained at least one hit for 33.1/59.8/ $85.0 / 95.3 \%$ within top $1 / 10 / 100 / 1000$ predictions, respectively. The naive post-filtering performed consistently worse than the base LZerD. An important conclusion from these results is that blind PPI site predictions cannot be used for improving docking prediction with the post-filtering procedure. On average it will only deteriorate prediction accuracy.
Unbound protein docking using actual PPI site prediction We have further benchmarked PI-LZerD on unbound docking cases. Out of 128 unbound docking cases in the protein-protein docking benchmark dataset 3.0 [87], 118 cases were selected that are not longer than 800 residues and have an MSA in the iPFAM database. The PPI sites were predicted by the meta-PPISP server. The iRMSD between bound and unbound complexes of this dataset ranges from $0.17 \AA$ to $8.38 \AA$ with an average of

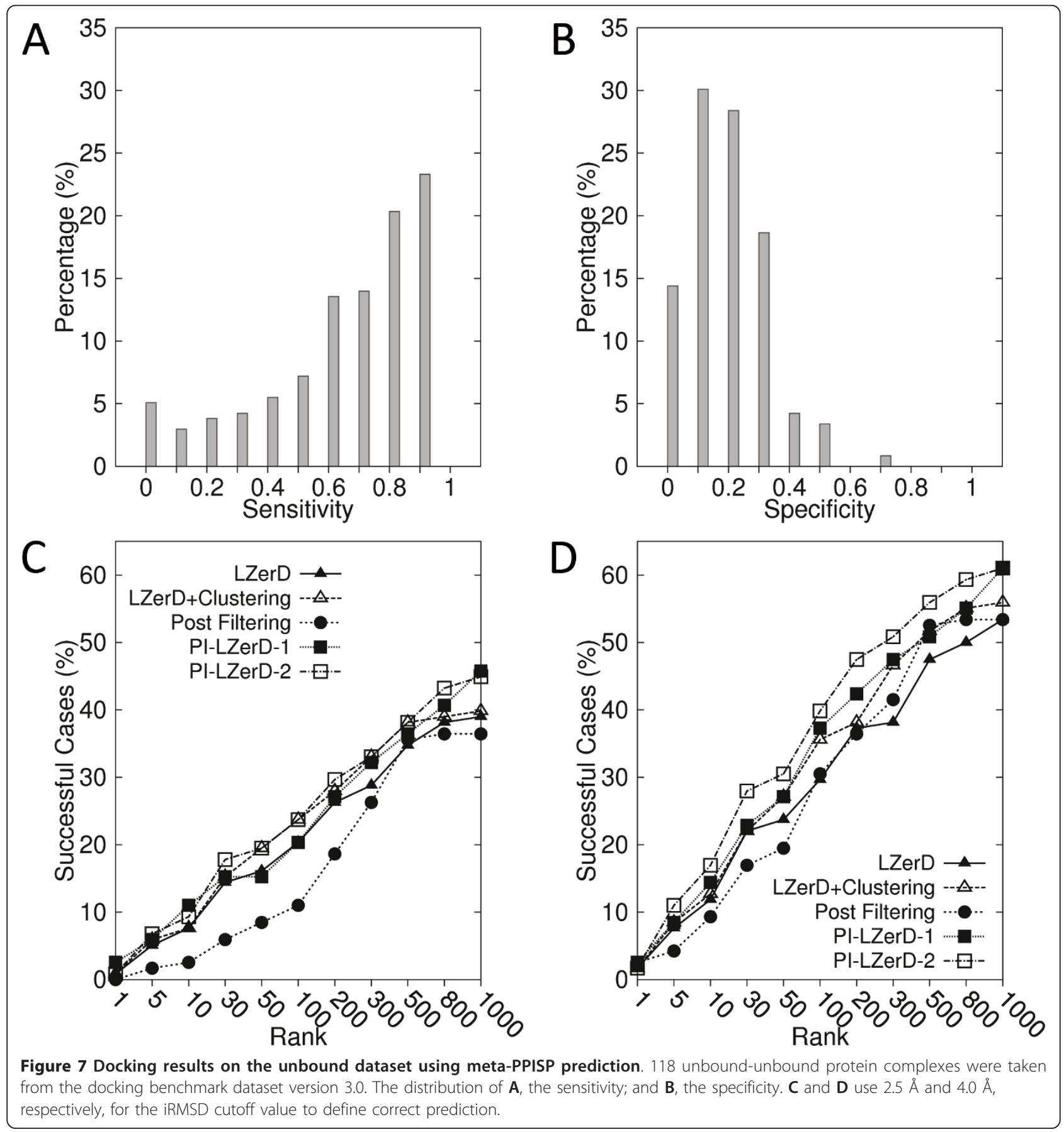


1.34 A. Figures 7A \&7B provide the distribution of the sensitivity and the specificity of the PPI site predictions by meta-PPISP. The average value was 0.684 and 0.231 for the sensitivity and the specificity, respectively.

We observe again the same trend as we observed in the previous experiments: PI-LZerD-2 showed consistently better success rate than the base LZerD at each rank cutoff (Figures 7C \&7D). At the rank cutoff of 10, 100, 1000, PI-LZerD-2 made successful predictions within $2.5 \AA$ iRMSD (Figure 7C) for 9.32\%, 23.73\%, and $44.92 \%$ of the cases, while the success rate of the base LZerD was $7.63 \%, 20.34 \%$, and $38.98 \%$. With $4.0 \AA$ iRMSD cutoff, (Figure 7D), the success rate of PILZerD-2/the base LZerD was 16.95/11.86, 39.83/29.66, and $61.02 / 53.39$ at 10,100, 1000 ranks. The naive postfiltering performed again worse than the base LZerD at most of the rank cutoff values.

The same prediction results are categorized according to the three difficulty levels for protein docking assigned by the docking benchmark dataset. The 118 unbound cases contain 87 rigid-body docking cases, 16 medium cases, and 15 difficult cases. In Figure 8, the success rates for the three difficulty levels are separately shown. For all the three levels, PI-LZerD consistently showed a higher or equal success rate as compared LZerD. The improvement by PI-LZerD over LZerD is more evident when $4.0 \AA$ iRMSD cutoff is used (Figure 8B).

Using this test set, we have also examined the effect of using a different number of decoys in the second round of LZerD run in PI-LZerD. As shown in the illustration of the PI-LZerD algorithm (Figure 2), we use top 30 lowest energy decoys and another 30 decoys with the largest clusters, thus 60 decoys, as the sources of updated PPI sites. We compared prediction results using 50 (i.e. 25 lowest energy decoys and 25 largest cluster decoys), 80, and 100 decoys in Additional file 1, Figure S5. The results show that using 60 docking decoys performs overall best among tested when the cutoff of $2.5 \AA$ is used. When the cutoff of $4.0 \AA$ is used to define near native decoys, all of them showed similar performance.

\section{Examples of docking prediction by PI-LZerD}

Here we show examples of docking predictions that illustrate difference of PI-LZerD as compared to the base LZerD and the naive post-filtering. The first two examples (Figures 9A \&9B) are from prediction using simulated PPI predictions. For all the cases actual PPI regions were shifted by 10 residues. The best iRMSD structures within top 50 decoys are shown.

First example is human cdk2 kinase complex with cell cycle-regulatory protein ckshs1 (PDB ID: 1BUH). The best predictions within top 50 using PI-LZerD/naive post-filtering/LzerD were $1.03 \AA$ (8)/9.09 ̊ (24)/9.91 $\AA$ (17) iRMSD, respectively. In the parentheses the rank of the decoys are shown. The second example (Figure 9B) is monoclonal antibody fab d44.1 complexed with lysozyme (1MLC). The best prediction using PI-LZerD-2/naive post-filtering/ LZerD were $0.89 \AA$ (34)/8.37 $\AA$ (9)/14.35 $\AA$ (22) iRMSD, respectively. The predicted ligand protein position by the naive post-filtering method (shown in red) indicates where the shifted PPI site information pointed. Thus, PI-LZerD managed to find the near native docking pose (green)
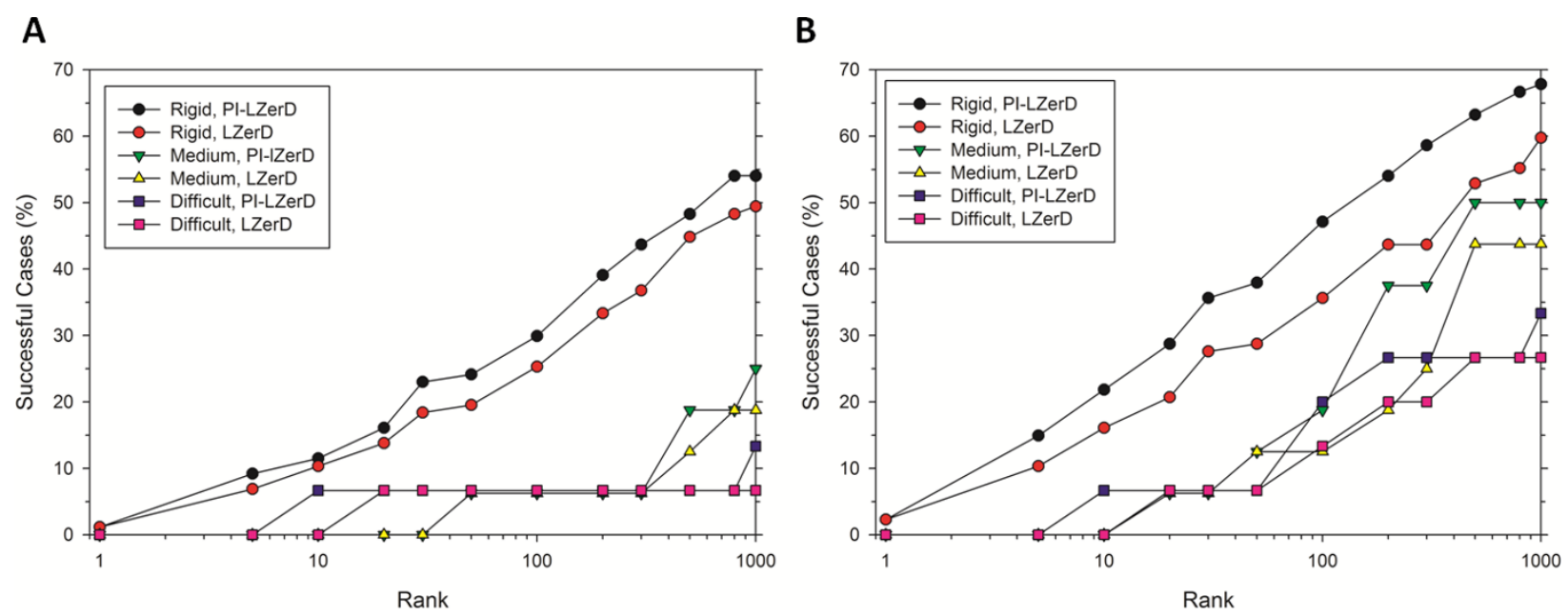

Figure 8 Docking results on the unbound dataset using meta-PPISP prediction are classified by the docking difficulty. The same results shown in Figure 7 are classified into three docking difficulty classes, rigid-body (87 cases), medium (16 cases), and, difficult (15 cases). A, B, results using $2.5 \AA$ and $4.0 \AA$, respectively, for the iRMSD cutoff value to define correct prediction. Black/red circles, the rigid-body category results by PI-LZerD/LZerD; green/yellow triangles, the medium category by PI-LZerD/LZerD; Black/red squares, the difficult category by PI-LZerD/ LZerD. 

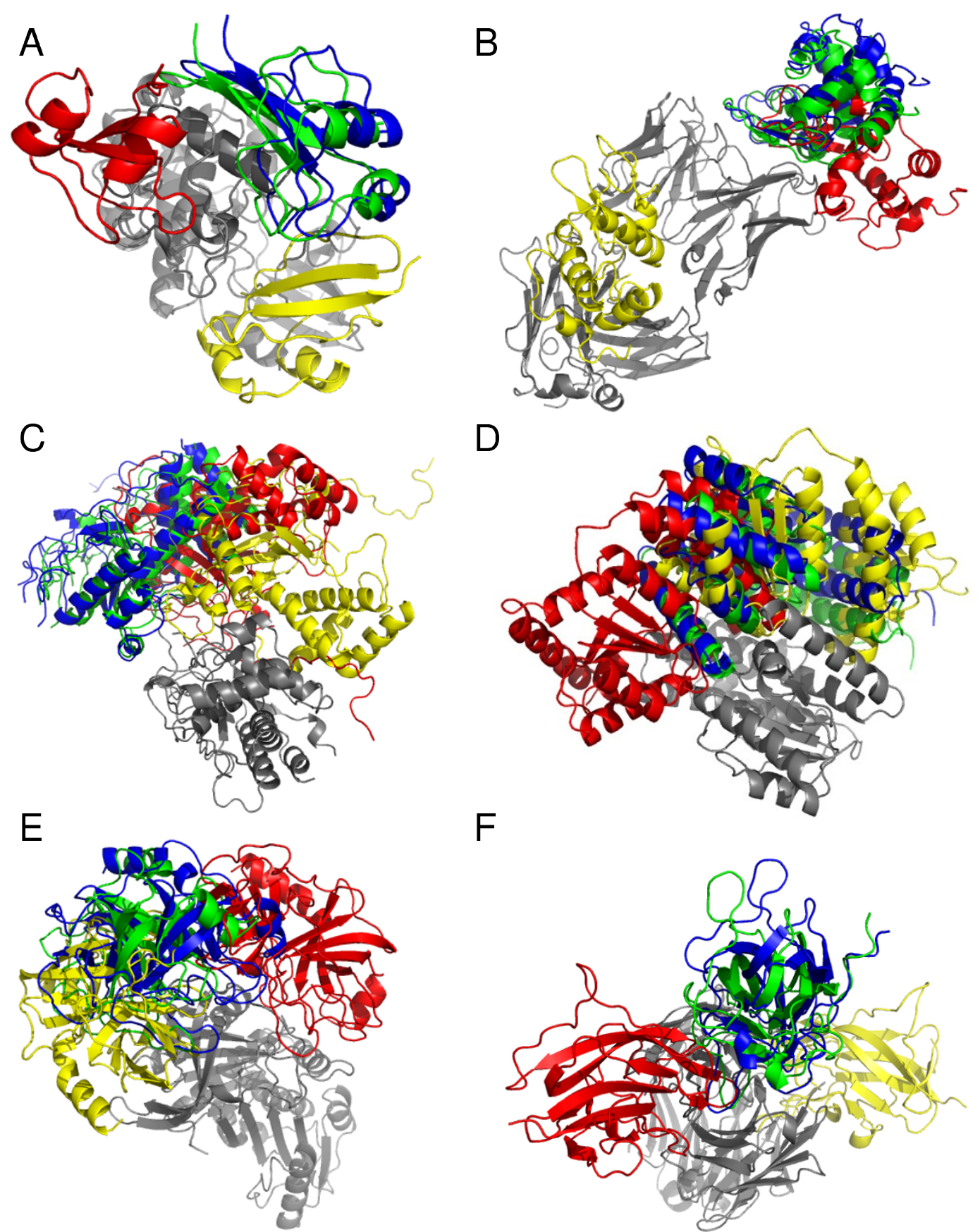

F

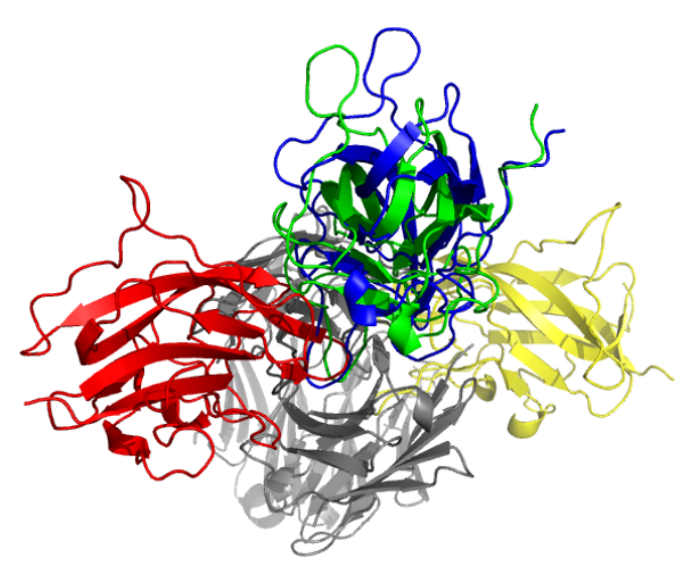

Figure 9 Examples of docking prediction. The best prediction within 50 ranks by PI-LZerD-2, the base LZerD, and naive post-filtering is shown in green, yellow, and red, respectively. The correct docking pose is shown in blue. Receptor proteins are shown in gray. First, predictions using 10 residue-shifted PPI site information are shown for $\mathbf{A}, 1 \mathrm{BUH}$; $\mathbf{B}, 1 \mathrm{MLC}$. For 1BUH, the iRMSD ( $\AA$ ) of prediction by PI-LZerD-2/post-filtering/base LZerD was $1.03 / 9.09 / 9.91$. The rank of the decoys was $8 / 24 / 17$. The sensitivity (= specificity, since the size of the shifted PPIs are same as the correct PPI) of the shifted PPI region was 0.33 for $1 \mathrm{BUHA}$ and 0.44 for $1 \mathrm{BUHB}$. For $1 \mathrm{MLC}$, iRMSD ( $\AA$ ) and the rank of the best prediction within 50 ranks by PI-LZerD-2/post-filtering/base LZerD were $0.89 \AA$ (34)/8.37 $\AA$ (9)/14.35 $\AA$ (22). The ranks are shown in the parentheses. The sensitivity (= specificity) of the shifted PPI region was 0.55 for chain $A$ and $B$, and 0.44 for chain E. C and $\mathbf{D}$ are predictions using actual PPI site predictions by meta-PPPISP for proteins from the iPFAM dataset. C, 1ADU. The iRMSD $(\AA)$ and the rank of the predictions by PI-LZerD-2/post-filtering/base LZerD were $1.04 \AA(48) / 14.90 \AA(37) / 10.85 \AA$ (43). The sensitivity/specificity of PPI site predictions were $0.77 / 0.47$ for 1 ADUA, and $0.00 / 0.00$ for 1ADUB. D, 1BMT. The iRMSD $(\AA)$ and the rank of the predictions by PI-LZerD-2/post-filtering/base LZerD were $2.31 \AA(36) / 14.44 \AA(31) / 13.02 \AA$ (44). The sensitivity/specificity of PPI site predictions were $0.11 / 0.06$ for 1BMTA, and 0.22/0.11 for 1BMTB. The last two examples are unbound docking cases with actual PPI predictions by meta-PPISP. E, 1OPH. The iRMSD ( $\AA$ ) and the rank of the predictions by PI-LZerD-2/post-filtering/ base LZerD were $3.76 \AA(42) / 5.71 \AA(39) / 10.28 \AA$ (16). The sensitivity/specificity of PPI site predictions were 1.00/0.32 for 1OPHA, and 0.70/0.18 for 1OPHB. F, 1IQD. The PI-LZerD-2/post-filtering/base LZerD: $2.91 \AA$ (23)/6.97 $\AA$ (16)/12.80 $\AA$ (32). The sensitivity/specificity of PPI site predictions were $0.53 / 0.16$ for $11 \mathrm{QDDA}$, and $0.89 / 0.20$ for $1 \mathrm{LQDB}$. 
from the originally provided wrong PPI site information. The near native pose (iRMSD $\leq 4.0 \AA$ ) was not found among the top 50 lowest energy score decoys.

The next two examples are taken from the iPFAM dataset where actual PPI predictions by meta-PPISP were used (Figure 6). Figure $9 \mathrm{C}$ is a complex of adenovirus single-stranded DNA-binding proteins (1ADU). The PPI site prediction by meta-PPISP is fine for one protein (sensitivity: 0.77 ) but totally missed the correct PPI site for another protein (sensitivity and specificity of 0.0 ). PILZerD-2 managed to identify a $1.04 \AA \AA$ iRMSD conformation (blue) while the naive post-filtering method made significantly wrong prediction (red). The LZerD energy function failed to identify the near native conformation within top 50 ranks (yellow). Figure 9D is a complex of methionine synthase (1BMT). The best PI-LZerD-2

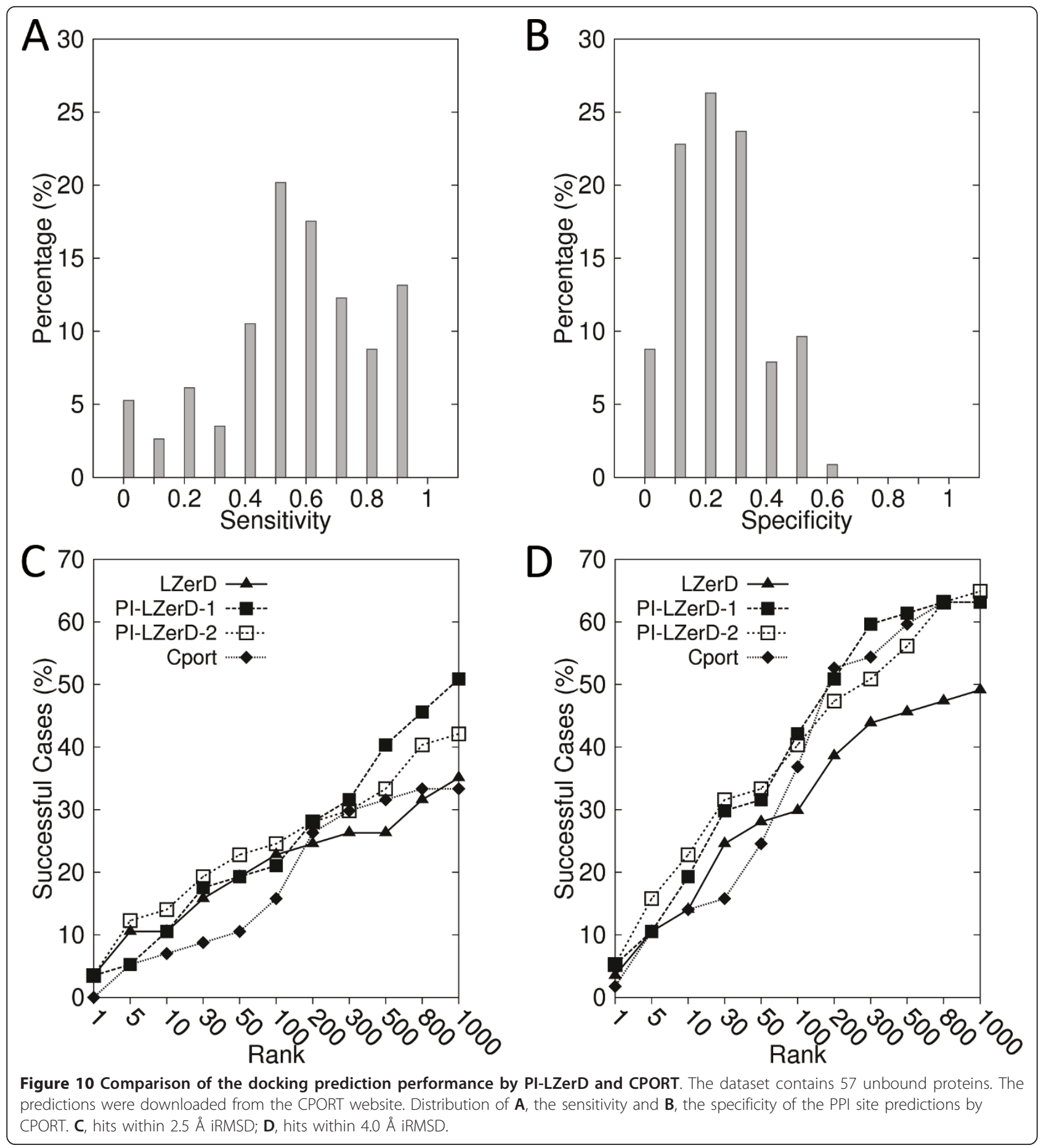


prediction is at $2.31 \AA$ iRMSD, while the post-filtering method and the base LZerD predictions are at iRMSD of 14.4 $\AA$ and $13.0 \AA$ iRMSD, respectively. The PPI prediction for the both chains are much worse than average.

The last two examples are from unbound docking experiments using meta-PPISP predictions. The first example is the predictions for $\alpha$-1-antitrypsin precursor and trypsinogen complex (1OPH). The best iRMSD predictions by PI-LZerD, the post-filtering, and base LZerD were $3.76 \AA$, $5.71 \AA$, and $10.28 \AA$, respectively. The last one, the complex of human factor VIII and human monoclonal BO2C11 Fab (1IQD), again PI-LZerD-2 identified a near-native pose (an iRMSD of $2.91 \AA$ ) (Figure $9 \mathrm{E}$ ). The base LZerD found lower energy decoys at very different position, an iMRSD of $10.28 \AA$.

\section{Comparison with an existing method}

Finally, we compare PI-LZerD with a recently published related method, CPORT [74]. CPORT takes a consensus approach for PPI site prediction, combining six web servers. Predicted PPI site information is used for proteinprotein docking in the framework the HADDOCK docking program. CPORT-HADDOCK translates predicted interface residues to what they call ambiguous interaction restraints (AIRs), which are distance restraints between provided (predicted) interface residues between a receptor and a ligand protein [14]. We used PPI site predictions of 57 unbound proteins that are made available as supplemental material of the paper at http://haddock.chem.uu.nl/services/CPORT. The distribution of the accuracy of the PPI site predictions is provided in Figures 10A \&10B.

The performance of docking prediction with CPORT and PI-LZerD are compared in Figures 10C \&10D. Overall, for both iRMSD threshold of $2.5 \AA$ (Figure $10 \mathrm{C}$ ) and $4.0 \AA$ (Figure 10D), PI-LZerD-2 showed a higher success rate at each rank cutoff ( $x$-axis). For example, PI-LZerD-2 obtained 14 success cases out of 57 complexes $(24.6 \%)$ within $2.5 \AA$ when top 100 scoring decoys are considered, while CPORT had 9 successful cases (15.8\%) at the same cutoff (Figure 9A). Using a 4.0 $\AA$ iRMSD threshold value, PI-LZerD-2 and CPORT obtained $23(40.4 \%)$ and 21 successful cases (36.8\%) within top 100 decoys, respectively.

\section{Conclusion}

We have developed PI-LZerD, a pairwise docking algorithm that uses imperfect PPI prediction to improve docking accuracy. In the series of experiments, we showed that PI-LZerD successfully improved docking results even when accuracy of PPI information is significantly low. Unlike the post-filtering whose success largely depends on the accuracy of provided PPI information, PI-LZerD can use imperfect PPI prediction to improve prediction by exploring docking poses in the neighborhood of provided PPI prediction. PI-LZerD identifies matches of two proteins at local surface regions that only partially overlap with the provided PPI prediction. In addition, employing two iterations of docking searches (PI-LZerD-2) is shown to be more effective than one round of docking (PI-LZerD-1) because the two iterations enable exploring further from the provided PPI site prediction. Improvement of the average docking accuracy by PI-LZerD over LZerD was observed consistently in the series of benchmark experiments including docking using actual PPI site predictions as well as unbound docking cases.

While this work focused on pairwise docking, the same procedure can be applied for multiple protein-protein docking algorithms [94-100]. As the protein interactions and their networks have become a very important research focus in systems biology, the procedure developed here will be valuable for providing physical picture of such interactions.

\section{Additional material}

\section{Additional file 1: Supplemental material for "Protein Docking Prediction Using Predicted Protein-Protein Interface". The file contains following five figures. Figure S1. Selecting decoys by the scoring function and/or by the cluster size. Figure S2. The procedure to compute "simulated" incorrect PPI site predictions. Figure S3. Docking prediction results using shifted PPI regions classified by the sensitivity of the PPI predictions. Figure S4. Docking prediction results using shifted PPI regions classified by the fnat of the PPI predictions. Figure S5. \\ Comparison of prediction results using different numbers of decoys for running the second iteration of LZerD.}

\section{Acknowledgements}

The authors gratefully acknowledge David La for helping preparing the benchmark dataset from the iPFAM database. We also thank Vishwesh Venkatraman and Yifeng D. Yang for providing the physics-based scoring function. We have used in part the Moffett clusters at Purdue University Rosen Center for Advanced Computing. This work has been supported by grants from the National Institutes of Health (R01GM075004, R01GM097528). DK also acknowledges grants from National Science Foundation (DMS0800568, EF0850009, IIS0915801).

\section{Author details}

'Department of Computer Science, Purdue University, West Lafayette, IN, 47907, USA. ${ }^{2}$ Department of Biological Science, Purdue University, West Lafayette, IN, 47907, USA. ${ }^{3}$ Markey Center for Structural Biology, Purdue University, West Lafayette, IN, 47907, USA.

\section{Authors' contributions}

BL participated in design, implemented the algorithms, and drafted the paper. DK conceived of the study, participated in its design, and finalized the manuscript. All authors read and approved the final manuscript.

Received: 26 August 2011 Accepted: 10 January 2012

Published: 10 January 2012

\section{References}

1. Aloy P, Russell RB: Ten thousand interactions for the molecular biologist. Nat Biotechnol 2004, 22:1317-1321. 
2. Russell RB, Alber F, Aloy P, Davis FP, Korkin D, Pichaud M, Topf M, Sali A: A structural perspective on protein-protein interactions. Curr Opin Struct Biol 2004, 14:313-324.

3. Szilagyi A, Grimm V, Arakaki AK, Skolnick J: Prediction of physical proteinprotein interactions. Phys Biol 2005, 2:S1-16.

4. Giot L, Bader JS, Brouwer C, Chaudhuri A, Kuang B, Li Y, Hao YL, Ooi CE, Godwin B, Vitols E, Vijayadamodar G, Pochart P, Machineni H, Welsh M, Kong Y, Zerhusen B, Malcolm R, Varrone Z, Collis A, Minto M, Burgess S, McDaniel L, Stimpson E, Spriggs F, Williams J, Neurath K, loime N, Agee M, Voss E, Furtak K, Renzulli R, Aanensen N, Carrolla S, Bickelhaupt E, Lazovatsky Y, DaSilva A, Zhong J, Stanyon CA, Finley RL, White KP, Braverman M, Jarvie T, Gold S, Leach M, Knight J, Shimkets RA, McKenna MP, Chant J, Rothberg JM: A protein interaction map of Drosophila melanogaster. Science 2003, 302:1727-1736.

5. Uetz P, Giot L, Cagney G, Mansfield TA, Judson RS, Knight JR, Lockshon D, Narayan V, Srinivasan M, Pochart P, Qureshi-Emili A, Li Y, Godwin B, Conover D, Kalbfleisch T, Vijayadamodar G, Yang M, Johnston M, Fields S, Rothberg JM: A comprehensive analysis of protein-protein interactions in Saccharomyces cerevisiae. Nature 2000, 403:623-627.

6. Ito T, Chiba T, Ozawa R, Yoshida M, Hattori M, Sakaki Y: A comprehensive two-hybrid analysis to explore the yeast protein interactome. Proc Natl Acad Sci USA 2001, 98:4569-4574.

7. Collura V, Boissy G: From protein-protein complexes to interactomics. Subcell Biochem 2007, 43:135-183.

8. Halperin I, Ma B, Wolfson H, Nussinov R: Principles of docking: An overview of search algorithms and a guide to scoring functions. Proteins 2002, 47:409-443.

9. Ritchie DW: Recent progress and future directions in protein-protein docking. Curr Protein Pept Sci 2008, 9:1-15.

10. Lensink MF, Wodak SJ: Docking and scoring protein interactions: CAPRI 2009. Proteins 2010, 78:3073-3084.

11. Gabb HA, Jackson RM, Sternberg MJ: Modelling protein docking using shape complementarity, electrostatics and biochemical information. $J$ Mol Biol 1997, 272:106-120.

12. Tovchigrechko A, Wells CA, Vakser IA: Docking of protein models. Protein Sci 2002, 11:1888-1896.

13. Gray JJ, Moughon S, Wang C, Schueler-Furman O, Kuhlman B, Rohl CA, Baker D: Protein-protein docking with simultaneous optimization of rigid-body displacement and side-chain conformations. J Mol Biol 2003, 331:281-99.

14. Dominguez C, Boelens R, Bonvin AM: HADDOCK: a protein-protein docking approach based on biochemical or biophysical information. $J$ Am Chem Soc 2003, 125:1731-1737.

15. Jiang F, Kim SH: "Soft docking": matching of molecular surface cubes. $J$ Mol Biol 1991, 219:79-102.

16. Chen R, Li L, Weng Z: ZDOCK: an initial-stage protein-docking algorithm. Proteins 2003, 52:80-87.

17. Ritchie DW, Kemp GJ: Protein docking using spherical polar Fourier correlations. Proteins 2000, 39:178-194.

18. Venkatraman V, Yang YD, Sael L, Kihara D: Protein-protein docking using region-based 3D Zernike descriptors. BMC Bioinformatics 2009, 10:407.

19. Garzon Jl, Lopez-Blanco JR, Pons C, Kovacs J, Abagyan R, Fernandez-Recio J, Chacon P: FRODOCK: a new approach for fast rotational protein-protein docking. Bioinformatics 2009, 25:2544-2551.

20. de Vries SJ, Bonvin AM: How proteins get in touch: interface prediction in the study of biomolecular complexes. Curr Protein Pept Sci 2008, 9:394-406

21. Kozakov D, Brenke R, Comeau SR, Vajda S: PIPER: an FFT-based protein docking program with pairwise potentials. Proteins 2006, 65:392-406.

22. Fischer D, Lin SL, Wolfson HL, Nussinov R: A geometry-based suite of molecular docking processes. J Mol Biol 1995, 248:459-477.

23. Gardiner EJ, Willett P, Artymiuk PJ: GAPDOCK: a Genetic Algorithm Approach to Protein Docking in CAPRI round 1. Proteins 2003, 52:10-14

24. Gardiner EJ, Willett $P$, Artymiuk PJ: Protein docking using a genetic algorithm. Proteins 2001, 44:44-56.

25. Li X, Moal IH, Bates PA: Detection and refinement of encounter complexes for protein-protein docking: taking account of macromolecular crowding. Proteins 2010, 78:3189-3196.

26. Schueler-Furman O, Wang C, Baker D: Progress in protein-protein docking: atomic resolution predictions in the CAPRI experiment using
RosettaDock with an improved treatment of side-chain flexibility. Proteins 2005, 60:187-194.

27. Shentu Z, Al HM, Bystroff C, Zaki MJ: Context shapes: Efficient complementary shape matching for protein-protein docking. Proteins 2008, 70:1056-1073.

28. Schneidman-Duhovny D, Inbar Y, Nussinov R, Wolfson HJ: PatchDock and SymmDock: servers for rigid and symmetric docking. Nucleic Acids Res 2005, 33:W363-W367.

29. Comeau SR, Gatchell DW, Vajda S, Camacho CJ: ClusPro: an automated docking and discrimination method for the prediction of protein complexes. Bioinformatics 2004, 20:45-50.

30. Kozakov D, Clodfelter KH, Vajda S, Camacho CJ: Optimal clustering for detecting near-native conformations in protein docking. Biophys $J$ 2005, 89:867-875.

31. Tong W, Weng Z: Clustering protein-protein docking predictions. Conf Proc IEEE Eng Med Biol Soc 2004, 4:2999-3002.

32. Das R, Andre I, Shen Y, Wu Y, Lemak A, Bansal S, Arrowsmith CH, Szyperski T, Baker D: Simultaneous prediction of protein folding and docking at high resolution. Proc Natl Acad Sci USA 2009, 106:18978-18983.

33. Shen Y, Paschalidis IC, Vakili P, Vajda S: Protein docking by the underestimation of free energy funnels in the space of encounter complexes. PLoS Comput Biol 2008, 4:e1000191.

34. Pierce $B$, Weng Z: ZRANK: reranking protein docking predictions with an optimized energy function. Proteins 2007, 67:1078-1086.

35. Hutchinson CL, Lowe PN, McLaughlin SH, Mott HR, Owen D: Mutational Analysis Reveals a Single Binding Interface between RhoA and Its Effector, PRK1. Biochemistry 2011.

36. Bradshaw RT, Patel BH, Tate EW, Leatherbarrow RJ, Gould IR: Comparing experimental and computational alanine scanning techniques for probing a prototypical protein-protein interaction. Protein Eng Des Sel 2011, 24:197-207.

37. Bogan AA, Thorn KS: Anatomy of hot spots in protein interfaces. J Mol Biol 1998, 280:1-9.

38. Delano WL: Unraveling hot spots in binding interfaces: progress and challenges. Curr Opin Struct Biol 2002, 12:14-20.

39. Dhungana S, Fessler MB, Tomer KB: Epitope mapping by differential chemical modification of antigens. Methods Mol Biol 2009, 524:119-134.

40. Speck SH, Koppenol WH, Dethmers JK, Osheroff N, Margoliash E, Rajagopalan KV: Definition of cytochrome $\mathrm{c}$ binding domains by chemical modification. Interaction of horse cytochrome $\mathrm{c}$ with beef sulfite oxidase and analysis of steady state kinetics. J Biol Chem 1981, 256:7394-7400.

41. Bonvin AM, Boelens R, Kaptein R: NMR analysis of protein interactions. Curr Opin Chem Biol 2005, 9:501-508.

42. Anand GS, Law D, Mandell JG, Snead AN, Tsigelny I, Taylor SS, Ten Eyck LF, Komives EA: Identification of the protein kinase A regulatory Rlalphacatalytic subunit interface by amide $\mathrm{H} / 2 \mathrm{H}$ exchange and protein docking. Proc Natl Acad Sci USA 2003, 100:13264-13269.

43. Meenan NA, Sharma A, Fleishman SJ, Macdonald CJ, Morel B, Boetzel R, Moore GR, Baker D, Kleanthous C: The structural and energetic basis for high selectivity in a high-affinity protein-protein interaction. Proc Natl Acad Sci USA 2010, 107:10080-10085.

44. Wiehe K, Pierce $B$, Tong WW, Hwang H, Mintseris J, Weng Z: The performance of ZDOCK and ZRANK in rounds 6-11 of CAPRI. Proteins 2007, 69:719-725.

45. Chelliah V, Blundell TL, Fernandez-Recio J: Efficient restraints for proteinprotein docking by comparison of observed amino acid substitution patterns with those predicted from local environment. J Mol Biol 2006, 357:1669-1682.

46. Pletneva EV, Laederach AT, Fulton DB, Kostic NM: The role of cation-pi interactions in biomolecular association. Design of peptides favoring interactions between cationic and aromatic amino acid side chains. J Am Chem Soc 2001, 123:6232-6245.

47. Jones S, Thornton JM: Analysis of protein-protein interaction sites using surface patches. J Mol Biol 1997, 272:121-132.

48. Lo CL, Chothia C, Janin J: The atomic structure of protein-protein recognition sites. J Mol Biol 1999, 285:2177-2198.

49. Liang S, Zhang C, Liu S, Zhou Y: Protein binding site prediction using an empirical scoring function. Nucleic Acids Res 2006, 34:3698-3707.

50. Jones S, Thornton JM: Principles of protein-protein interactions. Proc Natl Acad Sci USA 1996, 93:13-20. 
51. Neuvirth H, Raz R, Schreiber G: ProMate: a structure based prediction program to identify the location of protein-protein binding sites. $J \mathrm{Mol}$ Biol 2004, 338:181-199.

52. Negi SS, Braun W: Statistical analysis of physical-chemical properties and prediction of protein-protein interfaces. J Mol Model 2007, 13:1157-1167.

53. Mihalek I, Res I, Yao H, Lichtarge O: Combining inference from evolution and geometric probability in protein structure evaluation. $J \mathrm{Mol}$ Biol 2003, 331:263-279.

54. Zhou HX, Shan Y: Prediction of protein interaction sites from sequence profile and residue neighbor list. Proteins 2001, 44:336-343.

55. Tjong H, Qin S, Zhou HX: PI2PE: protein interface/interior prediction engine. Nucleic Acids Res 2007, 35:W357-W362.

56. Porollo A, Meller J: Prediction-based fingerprints of protein-protein interactions. Proteins 2007, 66:630-645.

57. Caffrey DR, Somaroo S, Hughes JD, Mintseris J, Huang ES: Are proteinprotein interfaces more conserved in sequence than the rest of the protein surface? Protein Sci 2004, 13:190-202.

58. Halperin I, Wolfson H, Nussinov R: Correlated mutations: advances and limitations. A study on fusion proteins and on the Cohesin-Dockerin families. Proteins 2006, 63:832-845.

59. Pazos F, Helmer-Citterich M, Ausiello G, Valencia A: Correlated mutations contain information about protein-protein interaction. J Mol Biol 1997, 271:511-523.

60. Pazos F, Valencia A: In silico two-hybrid system for the selection of physically interacting protein pairs. Proteins 2002, 47:219-227.

61. Kufareva I, Budagyan L, Raush E, Totrov M, Abagyan R: PIER: protein interface recognition for structural proteomics. Proteins 2007, 67:400-417.

62. Burgoyne NJ, Jackson RM: Predicting protein interaction sites: binding hot-spots in protein-protein and protein-ligand interfaces. Bioinformatics 2006, 22:1335-1342.

63. Liang S, Zhang J, Zhang S, Guo H: Prediction of the interaction site on the surface of an isolated protein structure by analysis of side chain energy scores. Proteins 2004, 57:548-557.

64. Bradford JR, Westhead DR: Improved prediction of protein-protein binding sites using a support vector machines approach. Bioinformatics 2005, 21:1487-1494.

65. Res I, Mihalek I, Lichtarge O: An evolution based classifier for prediction of protein interfaces without using protein structures. Bioinformatics 2005, 21:2496-2501.

66. Pettit FK, Bare E, Tsai A, Bowie JU: HotPatch: a statistical approach to finding biologically relevant features on protein surfaces. J Mol Biol 2007, 369:863-879.

67. Li MH, Lin L, Wang $X L$, Liu T: Protein-protein interaction site prediction based on conditional random fields. Bioinformatics 2007, 23:597-604.

68. La D, Kihara D: Predicting binding interfaces of protein-protein interactions. In Biological Data Mining in Protein Interaction Networks. Edited by: Li XL, Ng SK. Philadelphia: IGI-GLobal; 2010:64-79.

69. Ezkurdia I, Bartoli L, Fariselli P, Casadio R, Valencia A, Tress ML: Progress and challenges in predicting protein-protein interaction sites. Brief Bioinform 2009, 10:233-246.

70. Qin S, Zhou HX: meta-PPISP: a meta web server for protein-protein interaction site prediction. Bioinformatics 2007, 23:3386-3387.

71. Zhou HX, Qin S: Interaction-site prediction for protein complexes: a critical assessment. Bioinformatics 2007, 23:2203-2209.

72. Heuser $P$, Bau D, Benkert $P$, Schomburg D: Refinement of unbound protein docking studies using biological knowledge. Proteins 2005, 61:1059-1067.

73. Tress M, de JD, Grana O, Gomez MJ, Gomez-Puertas P, Gonzalez JM, Lopez G, Valencia A: Scoring docking models with evolutionary information. Proteins 2005, 60:275-280.

74. de Vries SJ, Bonvin AM: CPORT: A Consensus Interface Predictor and Its Performance in Prediction-Driven Docking with HADDOCK. PLOS ONE 2011, 6:e17695.

75. Huang $B$, Schroeder M: Using protein binding site prediction to improve protein docking. Gene 2008, 422:14-21.

76. Berman HM, Westbrook J, Feng Z, Gilliland G, Bhat TN, Weissig H, Shindyalov IN, Bourne PE: The Protein Data Bank. Nucleic Acids Res 2000, 28:235-242.

77. Wolfson H, Rigoutsos I: Geometric hashing: an overview. IEEE Computational Science Engineering 1997, 4:10-21.
78. Andrusier N, Mashiach E, Nussinov R, Wolfson HJ: Principles of flexible protein-protein docking. Proteins 2008, 73:271-289.

79. Meyer M, Wilson P, Schomburg D: Hydrogen bonding and molecular surface shape complementarity as a basis for protein docking. $\mathrm{J} \mathrm{Mol} \mathrm{Biol}$ 1996, 264:199-210

80. Lazaridis T, Karplus M: Effective energy functions for protein structure prediction. Curr Opin Struct Biol 2000, 10:139-145.

81. Eisenberg D, McLachlan AD: Solvation energy in protein folding and binding. Nature 1986, 319:199-203.

82. Zhang C, Vasmatzis G, Cornette $J$, DeLisi C: Determination of atomic desolvation energies from the structures of crystallized proteins. J Mol Biol 1997, 267:707-726.

83. Mintseris J, Wiehe K, Pierce B, Anderson R, Chen R, Janin J, Weng Z: Protein-Protein Docking Benchmark 2.0: an update. Proteins 2005, 60:214-216.

84. Huang SY, Zou X: An iterative knowledge-based scoring function for protein-protein recognition. Proteins 2008, 72:557-579.

85. Kabsch W: A discussion of the solution for the best rotation to relate two sets of vectors. Acta Cryst 1978, A34:827-828.

86. Janin J, Henrick K, Moult J, Eyck LT, Sternberg MJ, Vajda S, Vakser I, Wodak SJ: CAPRI: a Critical Assessment of PRedicted Interactions. Proteins 2003, 52:2-9.

87. Hwang H, Pierce B, Mintseris J, Janin J, Weng Z: Protein-protein docking benchmark version 3.0. Proteins 2008, 73:705-709.

88. Mendez R, Leplae R, Lensink MF, Wodak SJ: Assessment of CAPRI predictions in rounds 3-5 shows progress in docking procedures. Proteins 2005, 60:150-169.

89. Finn RD, Marshall M, Bateman A: iPfam: visualization of protein-protein interactions in PDB at domain and amino acid resolutions. Bioinformatics 2005, 21:410-412.

90. Finn RD, Mistry J, Tate J, Coggill P, Heger A, Pollington JE, Gavin OL, Gunasekaran P, Ceric G, Forslund K, Holm L, Sonnhammer EL, Eddy SR, Bateman A: The Pfam protein families database. Nucleic Acids Res 2010, 38:D211-D222

91. Uniprot Consortium: The Universal Protein Resource (UniProt) in 2010. Nucleic Acids Res 2010, 38:D142-D148.

92. Henrick K, Thornton JM: PQS: a protein quaternary structure file server. Trends Biochem Sci 1998, 23:358-361.

93. Edgar RC: MUSCLE: multiple sequence alignment with high accuracy and high throughput. Nucleic Acids Res 2004, 32:1792-1797.

94. Esquivel-Rodriguez J, Yang YD, Kihara D: Multi-LzerD: multiple protein docking for asymmetric complexes. 3DSIG 2011: Structural Bioinformatics and Computational Biophysics 2011.

95. Karaca E, Melquiond AS, de Vries SJ, Kastritis PL, Bonvin AM: Building macromolecular assemblies by information-driven docking: introducing the HADDOCK multibody docking server. Mol Cell Proteomics 2010, 9:1784-1794.

96. Comeau SR, Camacho CJ: Predicting oligomeric assemblies: N-mers a primer. J Struct Biol 2005, 150:233-244.

97. Berchanski A, Eisenstein M: Construction of molecular assemblies via docking: modeling of tetramers with D2 symmetry. Proteins 2003, 53:817-829.

98. Andre I, Bradley P, Wang C, Baker D: Prediction of the structure of symmetrical protein assemblies. Proc Natl Acad Sci USA 2007, 104:17656-17661.

99. Inbar $\mathrm{Y}$, Benyamini $H$, Nussinov R, Wolfson $\mathrm{HJ}$ : Combinatorial docking approach for structure prediction of large proteins and multi-molecular assemblies. Phys Biol 2005, 2:S156-S165.

100. Esquivel-Rodriguez J, Kihara D: Evaluation of multiple protein docking structures using correctly predicted pairwise subunits. BMC Bioinformatics 2011.

doi:10.1186/1471-2105-13-7

Cite this article as: Li and Kihara: Protein docking prediction using predicted protein-protein interface. BMC Bioinformatics 2012 13:7. 